\title{
Precise and Systematic Long-duration Study of Solar Modulation on Elementary Particles and Nuclei with AMS-02 on the Space Station
}

\author{
Maura Graziani*广 \\ KIT, Karlsruher Institut für Technologie \\ E-mail: maura.graziani@cern.ch
}

\begin{abstract}
We present the precise monthly time variation of the $e^{+}, e^{-}, p$ and $\mathrm{He}$ fluxes by the Alpha Magnetic Spectrometer on the International Space Station. The data were collected during the ascending phase of solar cycle \#24 through its maximum and toward its minimum. This covers one half solar cycle. For the first time, the time evolution of cosmic rays of opposite charges and different mass and charge are simultaneously measured during the reversal of the Sun's magnetic field polarity. These measurements are crucial for solar modulation modelling during periods of high-level solar activity. They also provide constraints for modelling astrophysical backgrounds for dark matter searches.
\end{abstract}

EPS-HEP 2017, European Physical Society conference on High Energy Physics 5-12 July 2017

Venice, Italy

* Speaker.

${ }^{\dagger}$ AMS Collaboration 


\section{Introduction}

Cosmic rays (CRs) are charged particles coming from outer space to the Earth's atmosphere with a broad range of energy that extends over several decades. Their origin, their transport in the Galaxy, and their interactions with the matter are not yet well known, so they are subject of extensive research. The experimental investigation of CRs composition and energy spectra is focused on seeking answers to fundamental science questions: the origin of CRs in the Galaxy, and the nature of cosmological dark matter (DM) particles.

However, low energetic $(E<20 \mathrm{GeV})$ CRs spectrum, when measured near Earth, is significantly affected by the solar activity and subject to the so-called solar modulation (SM) effect. The solar activity has a cycle which period is $\sim 11$ years, during which it increases reaching a maximum and then decreases again. The intensity of cosmic ray radiation is correlated (or rather anticorrelated) with the activity of the sun and as result, the measured spectrum at low energies is different from the Local Interstellare Spectrum (LIS), i.e. the spectrum outside the heliosphere.

FIg. 1 shows the current solar cycle 24, started on January 4, 2008 and reaching its maximum on April 2014 [4]. AMS-02 starts to take data on May 2011 and it will almost cover the whole solar cycle 24.

A deep understanding of the SM of CRs would allow to connect the spectrum observed near Earth to the true LIS. The SM can be studied measuring the fluxes as a function of the solar activity, i.e. of time. Charge-sign dependent effects of SM, more evident during the minimum of solar activity, are also expected.

Before PAMELA and AMS-02, the charge-sign dependent SM was mostly studied comparing "all electrons", which was actually the sum of $e^{-}$and $e^{+}$, with CRs protons and helium of the same rigidity. However, only simultaneous measurements of $e^{-}$and $e^{+}$(protons and anti-protons) over a complete solar activity cycle can represent a crucial test of the current charge-sign dependent modulation models. Precise and simultaneous measurements of CRs and their antiparticles by the same instrument are necessary. In this contest, AMS can provide the most accurate measurements of the time dependence of $e^{-}$and $e^{+}$fluxes since 2011 thanks to its high acceptance and the excellent performances of the detector. In August 2012 the spacecraft Voyager 1 has entered in the very local interstellar medium, crossing the solar modulation boundary, and measured for the first time the very local interstellar spectra at low energies, including the electron spectra between 5-20 MeV $[5,6,7,8]$. This data together with the solar modulated spectra measured near Earth, will allow to improve the knowledgement of the solar modulation effect on CR radiation.

\section{The detector}

AMS-02 is a multi-purpose CRs detector operating onboard the International Space Station (ISS) since May 2011. The instrument will be active for the entire ISS lifetime, i.e., until 2024 or beyond. With this long observation time and its large collecting power $\left(0.5 \mathrm{~m}^{2} \mathrm{sr}\right)$, AMS-02 is capable to provide high-quality data on $\mathrm{CR}$ fluxes at the $\mathrm{TeV}$ energy scale with unprecedent precision and sensitivity. Furthermore, the measurements of low energy CR fluxes over an entire 11-year solar cycle will enable us to perform a multichannel investigation of the solar modulation effect of Galactic CRs. The AMS-02 instrument is described in details in Ref. [1]. It is composed 
by several sub-detector systems that permit a redundant determination of the main characteristics of CR particles, i.e, arrival direction, particle identity (signed charge, mass) and its kinetic energy or rigidity $(R=p / Z)$. The particle direction and rigidity are obtained by the reconstruction of its trajectory along up to nine Silicon Tracker layers with $\sim 10 \mu m(\sim 30 \mu \mathrm{m})$ of spatial resolution on the $\mathrm{Y}(\mathrm{X})$ side. The velocity $\beta=v / c$ can be determined from the transit time between the upperTOF and lower-TOF scintillator planes along the track (for $Z=1, \Delta \beta / \beta 3 \%$ ), or more precisely using the RICH system (for $Z=1, \Delta \beta / \beta 10^{-3} \%$ ). The central part of AMS-02 is surrounded by an anti-coincidence system (ACC). The detector is completed with a Transition Radiation Detector (TRD), which is located at the top of the instrument, and a 18-layer electromagnetic calorimeter (ECAL), which is placed at the bottom. In the measurement of CR leptons, the signals in TRD and ECAL are used to discriminate the leptonic component from the hadronic background. The combination of these information allows for an efficient lepton/hadron separation power.

\section{Results}

Preliminary AMS-02 results for time variation of $e^{-}, e^{+}, p$ and $H e$ fluxes can be found in [3]. Long time variation effects can been observed. The intensity of the flux for all species decreases with time up to April 2014 that correspond to the maximum of solar activity. After this time, the flux intensity starts to increase again with time but for electrons. This is due to charge-sign dependent effects. These long time variation effects are due to the diffusion and magnetic drift of CRs in the heliosphere that, respectively, cause the anticorrelation between solar activity and flux intensity and the charge-sign dependent effects.

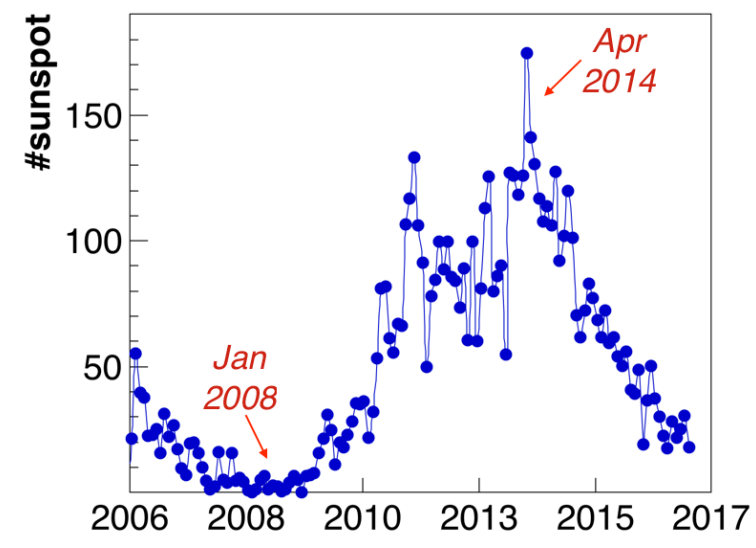

Figure 1: Solar cycle 24. It start on January 2008 and reaches its maximum on April 2014.

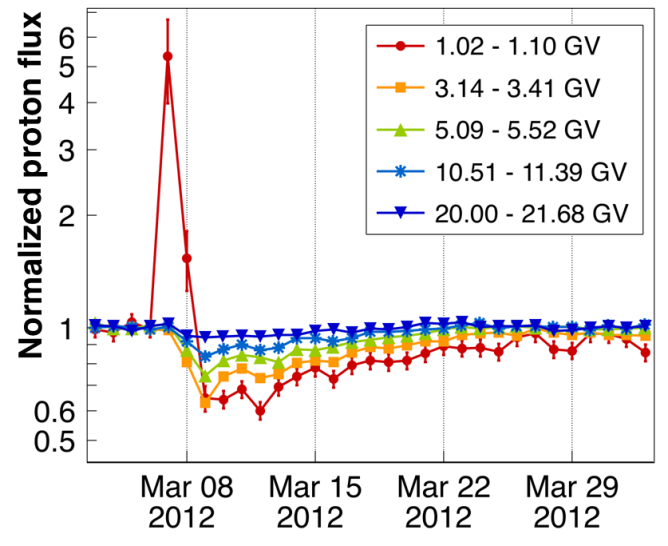

Figure 2: Normalized proton flux as a function of time for different rigidity bins, during the strongest SEP event occurred during the first 5 years of AMS-02 taking data period.

Togheter with the long time duration effects, small time structures can be observed. These structures are due to Coronal Mass Ejection (CME) from the sun. A CME is an unusual large release of plasma and magnetic field from solar corona that can causes SEP events, i.e. highenergetic particles coming from the sun, that increase the CRs intensity at low energies. SEP are 
of particular interest and importance because they can endanger life in outer space. The SEP is usually followed by a Forbush decrease (FD) that consists in a rapid decrease in CRs intensity due to the interaction with CME magnetic field. Fig. 2 shows the normalized proton flux as a function of time for different rigidity bins, during the strongest SEP event occurred during the first 5 years of AMS-02 taking data period. The CME effect on CRs intensity is clearly visible in the lowest rigidity bin as a SEP event (on March7, 2012), followed by a FD (on March 8, 2012).

\section{Conclusion}

A deep understanding of LIS is important for study in CRs background for DM searches. However, the measured CRs spectrum travels inside the heliosphere, it is affected by the activity of the sun and is different from the LIS. The study of CRs fluxes in time increases our knowledge of SM effects that should be taken into account to have a complete understanding of LIS. In this contest, AMS-02 can provide accurate measurements of CRs fluxes in time for several species: protons, helium, electrons, positrons. Some of these for the first time. The high performances of the instruments allows us to study the long scale time variations (anticorrelation, charge-sign dependencies) as well as short time variations (SEP, FD). Moreover, AMS-02 can provide the first accurate measurement of charge sign dependent effect for the same specie by mean of the observation of particle and antiparticle, like $e+/ e-$ and $\bar{p} / p$. The AMS-02 results for $e^{-}, e^{+}, p$ and $\mathrm{He}$ as a function of time, have been discussed. For all the species, the flux behavior consist in a first phase of decreasing and a second part of increasing that is consistent with the solar activity cycle. All particles with positive charge have their flux minimum during the maximum of solar activity while electron flux has its minimum later. This is due to solar modulation charge-sign dependent effects. Short time scale variations effects on CRs have also been observed.

\section{References}

[1] M. Aguilar et al., Phys. Rev. Lett., 110 (2013) 141102.

[2] Aguilar, M., Ali Cavasonza, L., Alpat, B., et al. Physical Review Letters, Vol.117, id.091103, 2016.

[3] Della Torre, S. and AMS-02 Collaboration. Results on Solar Physics from AMS-02, arXiv $.1612 .08441,2016$.

[4] http://www.solarham.net/

[5] D. A. Gurnett, W. S Kurth, L. F. Burlaga, and N. F. Ness. In situ observations of interstellar plasma with Voyager 1. Science, 341:1489-1492, 2013.

[6] E. C. Stone, A. C. Cummings, F. B. McDonald, B. C. Heikkila, N. Lal, andW. R.Webber. Voyager 1 Observes Low-Energy Galactic Cosmic Rays in a Region Depleted of Heliospheric Ions. Science, 341:150-153, 2013.

[7] W. R. Webber, P. R. Higbie, and F. B. McDonald. A comparison of the galactic cosmic ray H, He and $\mathrm{C} / \mathrm{O}$ nuclei spectra measured between 5 and $500 \mathrm{MeV} / n u c$ beyond $122 \mathrm{AU}$ at Voyager 1 with the predictions of a diffusion model for propagation in the galaxy. 2013.

[8] M.S. Potgieter. The charge-sign dependent effect in the solar modulation of cosmic rays. Advanced Space Research, 10.1016/j.asr.2013.04.015, 2014. 